\title{
LA AUDITORÍA MEDIO AMBIENTAL: Una especialidad necesaria de conocer y aplicar en el Perú
}

Dr. ERNESTO A. POLAR FALCÓN (*)

En la XXIII Conferencia Interamericana de Contabilidad, realizada en Puerto Rico en setiembre de 1999, en el Área 2-Auditoría, el único tema tratado en los trabajos profesionales presentados fue "La Auditoría Integral".

Los trabajos técnicos nacionales fueron diez, con los siguientes títulos:

- Auditoría Integral

- Auditoría Integral: precisiones sobre alcances y contenido

- La Auditoría Integral. Un salto al vacío

- La Auditoría Integral en el contexto de un mundo globalizado

- Auditoría Integral, amplitud conceptual, informe del auditor y problemas de implementación

- A Auditoría Integral en Entidade Binacional (En idioma portugués)

- Marco conceptual de la Auditoría Integral

- Marco de competencia de la Auditoría Integral: un nuevo rol para el profesional contable
- La Auditoría Integral. Un proceso de control gerencial

- La Auditoría Integral y el impacto ambiental

Los trabajos técnicos interamericanos fueron dos, con los siguientes títulos:

- Auditoría Integral, un enfoque integrador

- La Auditoría Integral: Un verdadero arquetipo de control empresarial

Se presentan estos listados de trabajos técnicos, con el propósito de incentivar su lectura, tanto a los Contadores Públicos como a los estudiantes universitarios que siguen la carrera de Contador Público. El objetivo es que tomen plena conciencia del futuro profesional de la Auditoría como una especialidad del Siglo XXI, llena de inquietudes y temas novedosos, que requieren estudio teórico intenso y aplicaciones prácticas en las diversas áreas económicas del Perú.

Como otra alternativa de lectura y de estudio profesional, también presentó dos temas interesantes en el Área 8 - Auditoría Interna, las cuales se muestran a continuación conjuntamente con los nombres de los títulos de trabajos interamericanos presentados:

(*) Docente Principal y Director de Asuntos Académicos de la Facultad de Ciencias Contables de laUNMSM 
La subcontratación de recursos externos en la función de Auditoría Interna:

\section{Dos trabajos técnicos nacionales:}

- La subcontratación de recursos externos en la función de Auditoría Interna

- Estrategia del Auditor Interno frente a la subcontratación de recursos externos

\section{Un trabajo técnico interamericano:}

- A Subcontratacao de Recursos Externos na Funcao de Auditoria Interna (En idioma portugués)

El rol del Auditor Interno en la Gestión de Riesgos y de Calidad:

\section{Tres trabajos técnicos nacionales:}

- Qualidade na Auditoria Integral (En idioma portugués)

- Aspectos metodológicos para una Auditoría a los sistemas de calidad

- El rol del Auditor Interno en la gestión de riesgos y de calidad

\section{Un trabajo técnico interamericano:}

- El Auditor Interno frente a la Gestión de Calidad y la Gestión de Riesgo

Se ha agregado el Área 8 - Auditoría Interna, por la importancia que le está dando la Asociación Interamericana de Contabilidad, ya que es una nueva área en el marco de las futuras Conferencias Interamericanas; y porque es la base profesional y de especialización contable de la Auditoría Medioambiental, tema importante de este artículo.
Los trabajos técnicos presentados en la XXIII Conferencia Interamericana de Contabilidad, tanto en el Área 1-Auditoría como en el Área 8 - Auditoría Interna, han sido analizados con la finalidad de localizar referencias a la Auditoría Medioambiental, tal como se describen a continuación:

1. En el trabajo técnico nacional titulado "Auditoría Integral: Precisiones sobre Alcance y Contenido" del chileno CP Jorge Alejandro Sánchez Henríquez, se encuentra el subtítulo "La Auditoría Integral y el Tema Medioambiental", que a continuación se transcribe literalmente:

"A finales del año pasado se realizaron las
XIX Jornadas de Ciencias Económicas del
Cono Sur, en ellas la Comisión de Auditoría
concluyó que "la profesión contable debe
contemplar la necesidad planteada en la
demanda de nuevos servicios profesio-
nales". Tuve la oportunidad de asistir a este
evento y entre otros, el tema ambiental era
dado como inherente a estos nuevos ser-
vicios que se incorporarían necesariamente
a este nuevo concepto de Auditoría Integral.

Para realizar una relación entre el tema ambiental y nuestra profesión, presentaré parte de lo planteado en el trabajo "Espacios de desarrollo y nuevos horizontes para la auditoría", en donde se hace un poco de historia y se aclara la relación entre la auditoría y el tema ambiental.

En 1946 se creó la International Organization for Standardization, con el propósito de desarrollar normas para la producción, el comercio y las comunicaciones, este organismo entre otras ha dictado las normas ISO 14000 referidas a temas ambientales.

La ISO 14010 define a la Auditoría Ambiental como el proceso documentado de verificaciones de obtención y evaluación objetiva de evidencia para determinar si actividades, eventos, condiciones y sistemas de gestión ambientales o información acerca de estas materias están acorde con criterios de auditoría y la comunicación de los resultados de este proceso al cliente. 
El 29 de junio de 1993 el Consejo de la Comunidad Europea adoptó la regulación 1836/93 orientada a la introducción de un "Esquema de Eco-Gestión y Eco-Auditoría". En Chile, en 1996, con motivo de la realización del XII Encuentro Nacional del Colegio de Contadores, Landa y Hernández plantean que la profesión de auditoría puede contribuir con su experiencia y servicios a las empresas y la Sociedad en progresar hacia el desarrollo sustentable y en la administración y contabilización de los aspectos relacionados con el medio ambiente. Agregan que la profesión tiene la fuerza, reputación y las herramientas necesarias para expandir este rol y contribuir en primer lugar al desarrollo de estándares y guías necesarias para auditar y revisar estados financieros relacionados con materias medioambientales, en segundo lugar, hacer los arreglos necesarios para obtener evidencias de auditoría que requiere la cooperación o uso del trabajo de otras profesiones o disciplinas en áreas fuera de la normal competencia de auditores financieros y en tercer lugar al desarrollo de estándares y guías necesarias para responder a las expectativas de los depositarios de nuevos servicios emergentes.

A este nivel de análisis podemos concluir que el tema ambiental no puede excluirse en el alcance de la Auditoría Integral, es más, cada vez se hace más difícil poder opinar globalmente de una empresa sin considerar los factores medioambientales que le afectan.

De acuerdo a un estudio realizado por Hugo Gubba y otros, presentado en las XVIII Jornadas de Ciencias Económicas del Cono Sur, en 5 países de América (Argentina, Brasil, Uruguay, Paraguay y Chile), se llegó a las siguientes conclusiones:

La existencia en toda la región de disposiciones legales y/o reglamentarias que regulan la actividad de las empresas o la implementación de proyectos con relación al medio ambiente.

La existencia de normas específicas relativas a la preparación de estados contables, o de la actuación profesional de estados contables, o de la actuación profesional en relación al mismo tema.

La escasa y en algunos casos nula uti- lización de procedimientos de auditoría de carácter específico.

La aceptación casi generalizada de la no contemplación en particular de este tipo de situaciones a nivel de los estados contables o de los informes de auditoría.

En mayo de 1995 el Comité Internacional de Prácticas de Auditoría (IAPC) de la Federación Internacional de Contadores (IFAC) emitió el draft (borrador de exposición) "La Profesión de Auditoría y el Medio Ambiente" que perseguía tres objetivos bien definidos. Primero generar ideas y punto de vista en torno a las materias a tratarse en la profesión de auditoría, con relación a la auditoría ambiental, segundo recolectar los puntos de vista de los profesionales sobre las implicancias más importantes de los temas ambientales en la auditoría de estados financieros y tercero promover la discusión entre los profesionales en cuanto a los reportes sobre el desempeño ambiental, la auditoría de tales informes y sobre posibles compromisos futuros, este documento sería un excelente punto de partida para definir técnicamente la inclusión de este tema como parte de la Auditoría Integral.

Los autores de temas interamericanos y nacionales de la XXII Conferencia Interamericana de Contabilidad mencionaron al medio ambiente como eventual componente de la Auditoría Integral, en este trabajo quiero ser categórico, en el sentido que un mercado globalizado como el actual, con clientes que están comenzando a manejar el concepto de valor agregado, el tema medioambiental no puede estar ausente, es más, hoy es requisito para ingresar a los mercados más importantes a nivel mundial"

2. En el trabajo técnico nacional titulado "La Auditoría Integral en el Contexto de un Mundo Globalizado" de los argentinos CP Armando Miguel Casal y CP Mario Wainstein, se ubica el subtítulo de "2. Los Problemas Medioambientales" que dice:

"Ciertas empresas han arrasado el delta de los ríos, dañando tierras, arroyos y la atmósfera a nivel de genocidio. Sin incluir los perjuicios a los habitantes que son de valor inestimable, los expertos en casos concretos calcularon en millones de dólares la indemnización mínima, que las compañías 
debían pagar por los males que la naturaleza había sufrido.

En muchas ocasiones en realidad no se pagaría nada y en cambio se compraría impunidad mediante acuerdos que liberarían de cualquier responsabilidad por los daños infligidos al medio ambiente. No obstante ello, se gastan mucho millones de dólares en campañas publicitarias que exaltan los desvelos por la defensa de la ecología.

También podemos citar las mayores catástrofes nucleares de la historia donde muchos murieron y muchos quedaron por siempre enfermos.

Según las proyecciones del Banco Mundial, las industrias ecologistas moverán fortunas mayores que la industria química.

La salvación del medio ambiente está siendo el más brillante negocio, en ciertas ocasiones, de las mismas empresas que lo aniquilan.

En un libro reciente, "The corporate planet", Joshua Karliner, San Francisco, 1997, brinda ejemplos ilustrativos y con valor pedagógico:

- Un grupo económico tiene cuatro empresas que más envenenan el aire del planeta, pero es también el mayor fabricante de equipos para el control de la contaminación.

- Una de las empresas con mayor responsabilidad en la generación de residuos industriales peligrosos en el mundo entero, ha desarrollado un sector de servicios especializados en la incineración y el entierro de residuos industriales peligrosos.

- Otro gigante multinacional, que vende armas nucleares, también vende millonarios equipos para limpiar su propia basura radioactiva.

En aras de la competitividad cada vez se solicitan más aplazamientos e incluso exenciones sobre las normas para la protección medioambiental.

La Conferencia de las Naciones Unidas sobre Medio Ambiente y Desarrollo (Río de Janeiro, junio de 1992), representa un aconte- cimiento histórico crucial, por cuanto fue la primera auténtica negociación global sobre la riqueza del mundo. De hecho, el objetivo consistía en plantear las condiciones y los medios para un desarrollo duradero de la economía mundial que fuera capaz de conciliar la elevada calidad de vida de los países más avanzados, con la solución de los problemas que afligen a miles de millones de seres humanos en los países pobres y poco desarrollados, y todo ello sin poner en peligro los equilibrios biológicos de la biosfera.

De esa Conferencia nació el Programa 21, que contiene el compromiso asumido por los gobiemos y los responsables políticos de más de 130 países en la "cumbre de la Tierra".

El Programa 21 abarca cuatro grandes áreas:

- La vertiente social y la economía

- La conservación y gestión de los recursos

- El reforzamiento del papel de los grandes grupos

- Los medios para asegurar la viabilidad del desarrollo

Y se complementa con:

- Una declaración de principios sobre los recursos forestales

- Un acuerdo marco de las Naciones Unidas sobre los cambios climáticos

- Un convenio sobre la diversidad biológica

- Una declaración general cubriendo todo el conjunto

Los problemas a que alude dicho programa son vitales, por ejemplo: combatir la pobreza, modificar los hábitos de consumo, proteger la salud humana, frenar la desertización, asegurar la viabilidad de la agricultura y el desarrollo rural, tratamiento de los desechos radioactivos, papel de la infancia y la juventud en lo tocante a un desarrollo duradero, reforzar el papel de los agricultores, reorientación de la industria y los negocios, etc. 
La política de "tareas comunes", consiste en acelerar la puesta en marcha de los compromisos y preceptos adoptados relacionados, tanto los candentes problemas de hoy, como de la necesidad de prepararse para los retos del próximo siglo.

El documento reconoce la responsabilidad de los gobiernos en la programación de un desarrollo duradero, a la vez que hace un llamamiento a favor de la más amplia participación popular para garantizar su aplicación y subraya la urgencia de nuevas y sustanciales ayudas financieras para los países subdesarrollados o emergentes".

3. En el trabajo técnico nacional titulado "Auditoría Integral, Amplitud Conceptual, Informe del Auditor y Problemas de Implementación", bajo el subtítulo 5. Informes presenta un "Modelo de Informe Anual de Auditoría Integral y el Punto" 5) Impacto ambiental dice:

"Según lo dictaminado por el Estudio Saturno de Ingeniería Ambiental, la empresa deberá asumir una fuerte inversión en los próximos cinco años para replantear totalmente el tratamiento de sus desechos industriales. Esta inversión deberá tenerse en cuenta en las proyecciones.

Y por último, el peruano CPC Jorge Luis Fox Santibáñez presentó el trabajo titulado "La Auditoría Integral y el Impacto Ambiental"; y en el subtítulo "Seguimiento Evolutivo del Comportamiento General en Relación con el Medio Ambiente" especifica lo siguiente:

\section{Causas}

La globalización de un fenómeno contemporáneo, que ha sentado sus bases a nuestro entender por dos factores relevantes que son:

1. El afán consumista; muchas veces la demanda generada por un determinado producto, ej. Aerosoles, biocidas, etc., no guarda relación a la necesidad real del mismo, la capacidad económica de algunos sectores fomenta un consumo desordenado demos- trando por una parte la ausencia de una cultura de consumo y por otra parte que la contaminación es un problema generado por todos.

2.La eliminación de fronteras; el avance tecnológico, la reducción de costos, la agresiva publicidad de crearnos necesidades, la ausencia de salvaguardas apropiadas ("ad valorem"), son factores que favorecen la producción indiscriminada de bienes los cuales obedecen a una demanda mundial sin contemplar los efectos contaminantes, ni el proceso de deterioro.

\section{Efectos y descripciones}

Lo que se ha dado en llamar "La manía del crecimiento" ha sido criticada duramente al haberse destacado los costos del crecimiento económico en relación con sus efectos sobre la calidad de vida. La contaminación se ha convertido, por desgracia en parte de la vida diaria.

Estudios realizados en el año 1982, en los EE.UU., determinaron que la contaminación de las aguas de ese país llegaba al 30\% (3).

Inseguridad Alimentaria, la afectación a sectores fértiles de una comunidad, genera que la producción se concentre en manos de pocos, en detrimento de otras regiones y de la seguridad alimentaria local.

(3) Francisco Mochón Morcillo. Catedrático de Ciencias Económicas y Empresariales UNED - Madrid.

\section{Afectación hidrobiológica}

El comportamiento indiferente hacia este sistema de vida, ha provocado lo siguiente:

- Destrucción de lagunas naturales

- Destrucción de manglares

- Destrucción de pantanos naturales

- Destrucción de lechos de algas

- Destrucción de arrecifes de coral

- Violación del espacio litoral 


\section{Afectaciones Oceanograficas}

Extinción en algunos casos de especies marinas de un determinado lugar por pescar con dinamita

Evacuación de residuos en altamar (detritos industriales)

Empleo de buques rastreros para la extracción de especies marinas, los volúmenes extraídos son tan relevantes que no da tiempo a la reproducción pertinente

Perforaciones submarinas para la extracción de petróleo y gas

Tránsito de navíos contaminantes violando el Convenio de MARPOL 73/74

Intoxicación de la cadena alimenticia de los mares por la evacuación de residuos compuestos por metales pesados, nitratos, fosfatos, plaguicidas, etc.

\section{Afectaciones Climáticas}

Producción y uso indiscriminado de aerosoles, ha provocado la destrucción de la capa de Ozono alterando en muchos casos las estaciones naturales

Aumento de la concentración de $\mathrm{C} 02$ generando gases de invernadero

\section{Afectaciones forestales}

La tala indiscriminada de árboles está incrementando la desertificación

Los huaicos son más frecuentes producto de la deforestación de zonas altas

Pérdida de la diversidad de tipos de vida salvaje y natural, producto de migración de especies

La expansión de la frontera agrícola por falta de conocimiento, contribuye de mala forma a la destrucción de este sistema de vida

\section{Afectaciones Hídricas}

La deforestación origina pérdida de la capacidad de los suelos en retener la hume- dad, derivando en sequías al no poderse generar reservas naturales de este elemento

- Deterioro de la calidad de las aguas de:

- Ríos

- Arroyos

- Lagos

- Mares

- Desaparición de especies acuáticas cuyo hábitat se torna inhabitable

- Las especies que han soportado estas inclemencias, ya no son aptas para el consumo humano y es un recurso menos para la sobre vivencia de una comunidad

\section{Afectaciones económicas}

- El efecto negativo es el sacrificio inmediato de un recurso, creyéndose que el beneficio a obtenerse va a ser equitativo lo cual es totalmente falso, sólo genera por un lado más pobreza y por otra más riqueza

- $\quad$ El factor condicionante que emplea el poder económico para seguir operando

- El despojo de los únicos bienes con que cuentan muchos países (riqueza natural)

Este trabajo técnico nacional es una excelente aportación profesional de un Contador Público, que seguramente como autor de este artículo y de muchos otros Contadores Públicos Peruanos, está preocupado por el constante crecimiento del deterioro medioambiental que se observa en el Perú y por la necesidad que se tiene de conocer más sobre la Auditoría Medioambiental y de su aplicación en trabajo profesional al respecto.

Entonces, es importante ampliar la base de conocimientos sobre la Auditoría Medioambiental. Al respecto, a continuación se presentan algunas definiciones de Auditoría Medioambiental:

1. Según la Cámara de Comercio Internacional, es "una herramienta de ges- 
tión que consiste en una evaluación objetiva, periódica, sistemática y documentada, que comprueba si la organización, gestión y equipo medioambiental están cumpliendo con el objetivo de salvaguardar el medio ambiente a través de: facilitar el control de gestión de las prácticas medioambientales y evaluar el nivel de cumplimiento de las políticas de la compañía incluyendo la adhesión a los requisitos de la normativa vigente.

2. La Agencia de Protección Medioambiental Estadounidense, la define como "la revisión periódica, documentada y sistemática que, desarrollada por entidades homologadas, sobre instalaciones y prácticas relacionadas con estándares medioambientales, tiende al cumplimiento de los siguientes objetivos: verificación del cumplimiento de estos estándares, evaluación de la efectividad de los sistemas de gestión medioambiental establecidos y evaluación de los riesgos naturales y de prácticas usuales".

3. El Reglamento Comunitario Español precisa que es "un instrumento de gestión que comprende una evaluación sistemática, documentada, periódica y objetiva de la eficiencia de la organización, el sistema de gestión y los procedimientos destinados a la protección del medio ambiente y que tiene por objeto: facilitar el control, por parte de la dirección, de las prácticas que puedan tener efectos sobre el medio ambiente y evaluar su adecuación a las políticas medioambientales de la empresa".

En todas estas definiciones se puede observar un paralelismo con relación a los requisitos de evaluación sistemática, periódica, documentada y objetiva que son exigidos a la actividad de auditoría y que parecen ir encaminados a asegurar la probabilidad del buen funcionamiento de los procedimientos que se aplican a cualquier tipo de auditoría. En función de estos términos se puede afirmar que "la auditoría medioambiental es una actividad desarrollada en intervalos de tiempo prefijados, ajustada a un conjunto de reglas o principios que, ordenadamente relacionados, contribuyen a la obtención de unos objetivos medioambientales establecidos, exenta de prejuicios y del modo de pensar de quien la realiza y, por último, basada en evidencias en las que apoyar sus conclusiones".

En base a las distintas clasificaciones de la auditoría interna y a las propias definiciones realizadas relativas a lo que se entiende por una auditoría medioambiental se puede aplicar la misma, fundamentalmente, desde dos puntos de vista: como auditoría de cumplimiento y como auditoría de gestión.

En la auditoría de cumplimiento, se recogerán aquellas actividades de supervisión tendientes a asegurar el cumplimiento de las leyes medioambientales en vigor, permitiendo así establecer cuáles son las condiciones de funcionamiento conformes a dichas normas, pudiendo definirse como aquella actividad en la que se comprueba si los gestores de una determinada entidad o institución pública han cumplido con las leyes, reglamentos y demás normas medioambientales que afectan el desarrollo de su actividad.

En la auditoría de gestión se incluyen aquellas actividades que permitirán ver cuál es la relación que se establece entre empresas-medioambiente, garantizando un control efectivo de las acciones desarrolladas, pudiendo definirse 
como aquella actividad tendiente a analizar y evaluar la política medioambiental de la entidad, global o parcialmente, su control medioambiental interno y sus procedimientos y sistemas de ecogestión, con el fin de emitir una opinión sobre la eficiencia de la utilización de los recursos y la eficacia en la consecución de los objetivos planificados.

Desde un punto de vista teórico, se puede agregar una clasificación más, que es la auditoría medioambiental estratégica, con las siguientes preocupaciones:

- Traducir la estrategia medioambiental de la organización en diferentes planes y programas.

- Tratar de analizar si la política medioambiental de la organización es adecuada para la consecución de los objetivos generales de la misma, así como para la estrategia global implantada.

- Garantizar la eficacia y eficiencia de los controles estratégicos establecidos.

Por lo expuesto, los elementos que se requieren incluir en el concepto de auditoría medioambiental son:

- Comprobar el cumplimiento actual con las leyes ambientales existentes, regulaciones y políticas organizativas.

- Confirmar la efectividad y correspondencia de la políticas medioambientales con la estrategia y objetivos generales de la organización.

- Evaluar la efectividad de los sistemas de control y gestión medioambiental establecidos en la organización.
- Determinar la operatividad de los negocios y los riesgos financieros derivados de las prácticas ambientales.

- Identificar y evaluar los problemas medioambientales a los que tradicionalmente se ha enfrentado la organización.

- Averiguar la responsabilidad potencial y los riesgos asociados a las transferencias de activos.

- Verificar si se divulga, y cómo se hace, la información referente a las relaciones entre empresa y medio ambiente, con el propósito de minimizar los problemas actuales y futuros.

- Detectar los costos medioambientales a los cuales se enfrenta la empresa, de forma que se traduzcan en una correcta fijación de precios.

- Proporcionar información útil a los directivos y funcionarios de la organización para la toma de decisiones.

Asimismo, la Auditoría Medioambiental, según la periodicidad en su implantación, se puede clasificar en auditorías periódicas y especiales.

Las auditorías medioambientales periódicas son las realizadas al amparo de un sistema de gestión medioambiental específico, implantado en la organización, cuyo objetivo es asegurar un correcto control del mismo para comprobar que su implementación es eficaz y eficiente. La periodicidad en la realización de las mismas dependerá, principalmente, del potencial contaminante de las actividades que están siendo objeto de dicha gestión medioambiental. A su vez, las mismas podrían distinguirse entre generales o integrales y sectoriales, contemplando las primeras todos los aspectos que interesan en la problemática o situación 
medioambiental y las segundas solamente algún aspecto concreto, como por ejemplo agua, residuos, ruido, energía, etc.

Las auditorías medioambientales especiales se desarrollarán en momentos concretos del tiempo, que no por ello son menos importantes, para algunas decisiones concretas, dependiendo de la situación e intereses de la empresa, y cuyo objeto es garantizar el cumplimiento de las normas medioambientales impuestas por la organización para el desarrollo de cualquier actividad y decisión concreta.

Las auditorías de gestión de la seguridad medioambientales especiales se consideran en las siguientes divisiones:

- Auditoría de gestión de la seguridad medioambiental.

- Auditoría medioambiental en la adquisición de empresas.

La auditoría de gestión de la seguridad medioambiental se desarrolla, como su propio nombre lo indica, cuando se trate de verificar los sistemas de seguridad frente a accidentes de carácter medioambiental, así como cuando se produzca un accidente de estas características en la organización, con el objetivo de determinar las causas de dicho accidente, el control de los sistemas de emergencia establecidos en la entidad y la definición de acciones correctoras y preventivas.

La auditoría medioambiental en la adquisición de empresas se desarrolla cuando se produce la adquisición, absorción o fusión de otra organización y tratan de analizar el actual nivel medioambiental de la organización con la que se va a integrar, con el objeto de determinar los riesgos medioambientales que se están asumiendo al tomar dicha decisión.
Todas estas definiciones, clasificaciones y enunciados se han obtenido analizando parcialmente la bibliografía que se adjunta a este artículo. Se agregan los comentarios y las adecuaciones efectuadas por el autor de este artículo, en función a su experiencia en Auditoría Financiera, especialidad en ejercicio profesional vigente.

Asimismo, se puede observar que la Auditoría Medioambiental, ya finalizando el Siglo XX contaba con excelente bibliografía, sin mucha difusión en el Perú; por lo que es necesario conocerla y adquirir dichos libros.

Hasta aquí se ha presentado a la Auditoría Medioambiental, desarrollada como una clasificación más de la Auditoría Integral. Ahora, es necesario agregar que también se aplica a la Auditoría Financiera, por cuanto al emitir un Dictamen, se tiene que tomar en cuenta aquellos aspectos medioambientales que contienen los Estados Financieros preparados por una empresa; debiendo preocuparse si se está ocultando o valorando a menores montos, las provisiones y contingencias de carácter medioambiental; sí están adecuadamente presentadas las inversiones relacionadas con el medio ambiente en el cual opera la empresa y las obligaciones contraídas al respecto; así como los costos y gastos que se han incurrido en las compras de bienes y servicios que de una u otra manera afectan el medio ambiente.

En el caso de que el auditor considere oportuno puede agregar en su Dictamen algunas salvedades relativas a la información medioambiental que presentan los Estados Financieros, llegando el caso extremo de emitir un Dictamen con opinión desfavorable, cuando existe extrema gravedad en el deterioro del medio ambiente. 


\section{CONCLUSIONES}

1.- Es evidente que la Auditoría Medioambiental en el Perú todavía no tiene la suficiente difusión profesional, ni está considerada como un capítulo más de estudio en los cursos de Auditoría Integral, que ya están aplicando algunas Universidades Peruanas.

2.- Que es necesario mostrar a los empresarios y directores de empresas de la importancia que tiene la Auditoría Medioambiental al informar sobre los daños que se presentan en el medio ambiente operativo, tomando en cuenta las recomendaciones que contienen dichos informes y procediendo a aplicarlas de inmediato.

3.-En la búsqueda de información sobre Auditoría Medioambiental para el presente artículo se ha descubierto que existe excelente bibliografía, que es necesario obtenerla para el estudio profesional y universitario, en el marco de la investigación científica y tecnológica, con la finalidad de formar investigadores dedicados exclusivamente a esta especialidad de la profesión del Contador Público.

4.- Que las definiciones, clasificaciones y otras áreas de la Auditoría Medioambiental mostradas en el presente artículo, representan una mínima expresión inicial de esta especialidad, frente al potencial de temas desarrollados en países preocupados por los problemas medioambientales que tienen; por lo que es prioridad atender la demanda que se hace para programar una serie de acciones que conduzcan al conocimiento de tal potencial profesional acumulado y su aplicación a la problemática medioambiental del Perú en las actividades pesqueras, agroindustriales, mineras, petroleras, que actualmente están produciendo enorme deterioro medio ambiental en perjuicio de sus habitantes y de la conservación de los recursos naturales que posee.

\section{BIBLIOGRAFIA}

CÁMARA DE COMERCIO INTERNACIONAL "Guía de la Cámara de Comercio Internacional para un proceso de auditoría medioambiental eficaz".Edición ICC Publishing.

CONESA FERNÁNDEZ, Vítora, V.

"Auditorías medioambientales - Guía Metodológica". Ediciones Mundi Prensa, España.

ESCANCIANOMONTOUSSE, L.

"La auditoría técnica". Edición P. Doble, España.

MARTÍN MATEO, $\mathbf{R}$.

"Nuevos instrumentos para la tutela ambiental". Edición Trivium, España.

MIÑANA SANJUANBENITO, F.

"Objetivos y fases de una auditoría medioambiental". Tesis de Maestría, España.

SEOÁNEZCALVO,M.

"Auditorías medioambientales y gestión medioambiental en la empresa". Ediciones Mundi, España.

TEJADA PONCE, A.

"La gestión y el control socio-económico de las interacciones Empresa-Medio Ambiente -Contribuciones de la contabilidad a la gestión sostenible de las empresas". Tesis Doctoral. 